\section{Observing brownian motion in vibration-fluidized granular matter}

\section{G. D’Anna ${ }^{1}$, P. Mayor ${ }^{1}$, A. Barrat ${ }^{2}$, V. Loreto ${ }^{3}$ \& Franco Nori ${ }^{4,5}$}

${ }^{1}$ Institut de Physique de la Matière Complexe, Faculté des Sciences de Base, Ecole Polytechnique Fédérale de Lausanne, CH-1015 Lausanne, Switzerland

${ }^{2}$ Laboratoire de Physique Théorique, Unité Mixte de Recherche UMR 8627, Bâtiment 210, Université de Paris-Sud, 91405 Orsay Cedex, France

${ }^{3}$ Università degli Studi di Roma La Sapienza, Dipartimento di Fisica, and INFM, Center for Statistical Mechanics and Complexity, Piazzale A. Moro 5, 00185 Rome, Italy

${ }^{4}$ Frontier Research System, The Institute of Physical and Chemical Research (RIKEN), Wako-shi, Saitama 351-0198, Japan

${ }^{5}$ Center for Theoretical Physics, Department of Physics, CSCS, University of Michigan, Ann Arbor, Michigan 48109-1120, USA

Observation of the rotational brownian motion ${ }^{1,2}$ of a very fine wire immersed in a gas led to one of the most important ideas of equilibrium statistical mechanics. Namely, the many-particle problem of a large number of molecules colliding with the wire can be represented by just two macroscopic parameters: viscosity and temperature. Interest has arisen in the question of whether this idea (mathematically developed in the Langevin model and the fluctuation-dissipation theorem ${ }^{3,4}$ ) can also be used to describe systems that are far from equilibrium. Here we report an experimental investigation of an archetypal non-equilibrium system, involving a sensitive torsion oscillator immersed in a granular system ${ }^{5,6}$ of millimetre-size grains that are fluidized by strong external vibrations. The vibro-fluidized granular medium is a driven environment, with continuous injection and dissipation of energy, and the immersed oscillator can be seen as analogous to an elastically bound brownian particle. By measuring the noise and the susceptibility, we show that the experiment can be treated (to a first approximation) with the equilibrium formalism. This gives experimental access to a granular viscosity and an effective temperature; however, these quantities are anisotropic and inhomogeneous. Surprisingly, the vibrofluidized granular matter behaves as a 'thermal' bath satisfying a fluctuation-dissipation relation.

Before describing the experiment in the granular medium we briefly recall the Langevin formalism for the brownian motion of a torsion oscillator immersed in a usual liquid at temperature $T$. This is a canonical problem of statistical mechanics ${ }^{4}$. The Langevin equation of the oscillator is $I\left(\mathrm{~d}^{2} \theta / \mathrm{d} t^{2}\right)+\alpha(\mathrm{d} \theta / \mathrm{d} t)+G \theta=C_{\text {ext }}(t)+$ $R(t)$, where $\theta$ is the angular deflection, $I$ is the moment of inertia of the oscillator, $\alpha$ the friction coefficient determining the viscous torque, $G$ the fibre elastic torsion constant, $C_{\text {ext }}(t)$ the external torque, and $R(t)$ a randomly fluctuating torque, assumed to be a gaussian white noise of zero mean. This equation is valid on timescales large compared to the correlation time of the random torque.

The Langevin equation can be solved by Fourier transformation. Without the external torque $\left(C_{\text {ext }}(t)=0\right)$, using the WienerKhintchine theorem for stationary processes ${ }^{4}$, one obtains the noise power spectral density (PSD), that is, twice the Fourier transformation of the auto-correlation function $\left\langle\theta(t) \theta\left(t^{\prime}\right)\right\rangle$, which gives $S(\omega)=2 q /\left[I^{2}\left(\omega_{\mathrm{o}}^{2}-\omega^{2}\right)^{2}+\alpha^{2} \omega^{2}\right]$ where $\omega_{\mathrm{o}}=\sqrt{G / I}$ is the natural pulsation of the oscillator and $2 q$ is the PSD of the random torque $R(t)$. Moreover, one has the relation $q=2 \alpha k_{\mathrm{B}} T$, which guarantees that the system is in thermodynamic equilibrium and that the equipartition of energy holds. On the other hand, solving the Langevin equation with the external torque $C_{\text {ext }}(t)$, and focusing on timescales large compared to the correlation time of the random torque, yields the complex susceptibility $\chi(\omega)=\theta(\omega) / C_{\text {ext }}(\omega)=$ $\chi^{\prime}(\omega)-i \chi^{\prime \prime}(\omega)$ with $\chi(\omega)=1 /\left[I\left(\omega_{o}^{2}-\omega^{2}\right)+i \alpha \omega\right]$. One finds that the ratio $S(\omega) \omega /\left(4 \chi^{\prime \prime}(\omega)\right)=k_{B} T$ is proportional to the temperature of the liquid, and is independent of the oscillator characteristics, such as its mass or shape. This represents a formulation of the fluctuation-dissipation (FD) theorem ${ }^{4}$ (FDT), expressed in the frequency space. Summing up, measuring the susceptibility and the noise PSD of the brownian motion of the oscillator allows one to consistently determine the viscosity and the temperature of the liquid, seen as an ideal thermal bath at equilibrium.

The natural question that arises at this point is how much of this formalism survives if the experiment is performed in a strongly non-equilibrium system, notably in a vibro-fluidized granular medium $^{5,6}$. A granular system is an assembly of particles, such as sand grains or glass beads, interacting by contact forces and featuring a very large number of macroscopic degrees of freedom, corresponding in first approximation to the positions and velocities of all grains. However, such a system is not in 'equilibrium' in the thermodynamic sense, since the thermal energy $k_{\mathrm{B}} T$ at room temperature is too small to induce any macroscopic grain fluctuations. Nevertheless, owing to the very large number of degrees of freedom, one expects that an analogy to the thermally induced brownian motion is possible when the system is externally driven and grain motion occurs by continuous injection of energy.

We have realized the experiment by immersing a torsion oscillator in a granular medium composed of glass beads (Fig. 1). The container, filled with the glass beads, is continuously shaken by a vertical vibrator, with a high-frequency filtered white noise, cut off below $300 \mathrm{~Hz}$ and above $900 \mathrm{~Hz}$. Notice the important point that a filtered white noise is used in order to obtain a homogeneous and continuous agitation of the granular medium, discarding undesired effects such as pattern formation, rolls, and other instabilities in the granular motion ${ }^{5}$. The purpose is not to provide $a b$ initio a random

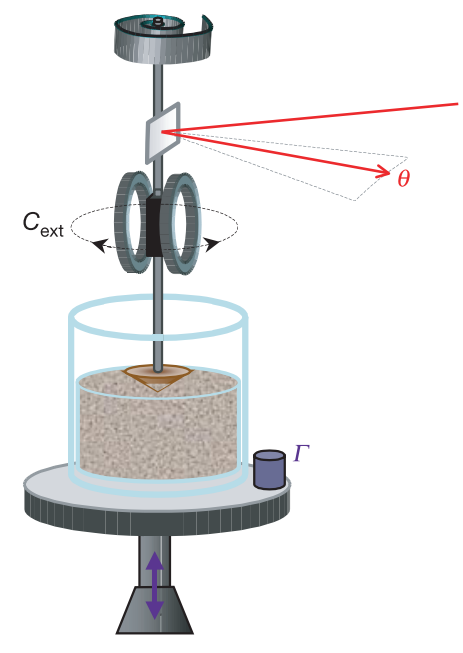

Figure 1 Sketch of the torsion oscillator immersed in a 'viscous liquid'. Here the 'viscous liquid' is a granular medium fluidized by strong external vibration (see text for details). The granular material consists of glass beads of diameter $1.1 \pm 0.05 \mathrm{~mm}$ contained in a metallic bucket of $60 \mathrm{~mm}$ height and $94 \mathrm{~mm}$ diameter, filled to a height of $31 \mathrm{~mm}$. The oscillator has a natural frequency of about $12 \mathrm{~Hz}$, depending on the probe used. The main probe used is a cone of apex angle $120^{\circ}$, covered with a single layer of glass beads, glued on by epoxy adhesive. Probes with different shapes have also been used, in particular cylindrical probes with various diameters (see Fig. 3). The probe is immersed to depth $L$. The oscillator allows measurement of both the noise PSD and the complex susceptibility. An accelerometer permits measurement of the intensity of the external vibration, $\Gamma$. Notice that the oscillator does not move in the vertical or horizontal directions. For this reason, below $\Gamma \approx 1$ the oscillator acts as a localized perturbation point, and the study of the granular dynamics at low $\Gamma$ requires a different approach ${ }^{14,15}$. 
torque with white noise spectrum to the oscillator. Indeed, we observe the motion of the oscillator in a low-frequency range (10$50 \mathrm{~Hz}$ ) compared to the applied vibration high-frequency range. The torsion oscillator probe, with various moments of inertia and shapes, is immersed at a depth $L$ from the granular surface. The oscillator is otherwise isolated from the container and the vibrator, and does not move in the vertical or horizontal directions. An accelerometer on the container is used to measure the acceleration spectrum, normalized to the acceleration of gravity, $A(\omega)$. We quantify the 'intensity' of the external shaking by $\Gamma$, defined as the square root of the band power of $A(\omega)$ in the range of about $1 \mathrm{~Hz}$ to $10 \mathrm{kHz}$, that is, $\Gamma^{2}=\int A(f) \mathrm{d} f$ integrated in the above frequency range, with $\omega=2 \pi f$. In the case of a sinusoidal vibration of amplitude $a_{\mathrm{s}}$ and frequency $f_{\mathrm{s}}=\omega_{\mathrm{s}} / 2 \pi$, the normalized acceleration spectrum is $A(f)=\left[a_{\mathrm{s}}\left(2 \pi f_{\mathrm{s}}\right)^{2} / g\right]^{2} \delta\left(f-f_{\mathrm{s}}\right)$ and one recovers the usual definition of the vibration intensity, that is, $\Gamma=a_{\mathrm{s}} \omega_{\mathrm{s}}^{2} / g$. For sinusoidal vibrations, $\Gamma=1$ is the lift-on threshold above which a single grain starts to 'fly'. In this work we employ vibration intensities up to $\Gamma \approx 15$.

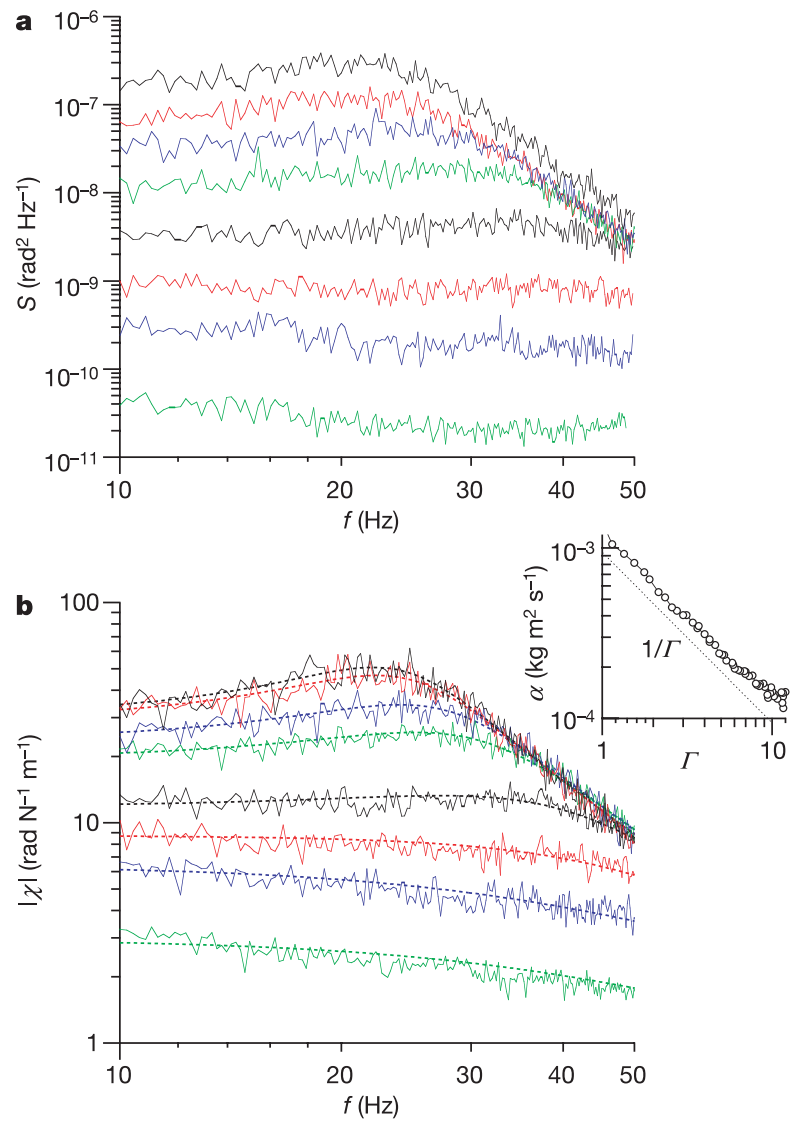

Figure 2 Noise and susceptibility for different vibration intensities. The probe is conical, immersed to about $11 \mathrm{~mm}$ from the surface. a, Noise PSD, $S(\omega)$, versus the frequency, $f$ for different vibration intensities $\Gamma$. The intensity values are, from top to bottom, 11.6, $10.0,7.3,5.4,3.7,2.2,1.5$ and 1.0 . b. Modulus of the complex susceptibility $|\chi(\omega)|$ versus the frequency $f=\omega / 2 \pi$, for different vibration intensities $\Gamma$. The intensity values are as above. Each curve (continuous line) is fitted (dashed line) with the expression $|\chi(\omega)|=\left[l^{2}\left(\omega_{0}^{2}-\omega^{2}\right)^{2}+\alpha^{2} \omega^{2}\right]^{-1 / 2}$, with two free parameters, $\omega_{0}$ and $\alpha$. The moment of inertia of the oscillator is $I=1.5 \times 10^{-6} \mathrm{~kg} \mathrm{~m}^{2}$, and the applied torque amplitude is $C_{\mathrm{e}}=3.2 \times 10^{-5} \mathrm{~N} \mathrm{~m}$. The parameter $\omega_{0}$ shows a small dependence on $\Gamma$ : it features a small increase from $12 \mathrm{~Hz}$ to $18 \mathrm{~Hz}$ by reducing $\Gamma$. The inset shows the friction coefficient $\alpha$, obtained from the fit to the curves $|\chi(\omega)|$ in the main panel, versus $\Gamma$. The dotted line is a power law $\alpha \propto 1 / \Gamma$. The $1 / \Gamma$ dependence is also systematically observed using susceptibility data obtained with probes of different shapes.
We now use the oscillator to measure (1) the noise PSD, and (2) the susceptibility of the vibro-fluidized granular medium. (1) In the absence of the external torque $\left(C_{\text {ext }}(t)=0\right)$ the immersed oscillator performs an irregular free angular motion, induced by the continuous interactions of the grains against the probe. The angular deflections $\theta$ are detected optically using a mirror fixed on the oscillator and, from the time-series of the angular deflections, $\theta(t)$, we obtain the noise PSD, $S(\omega)$, as shown in Fig. 2a for several values
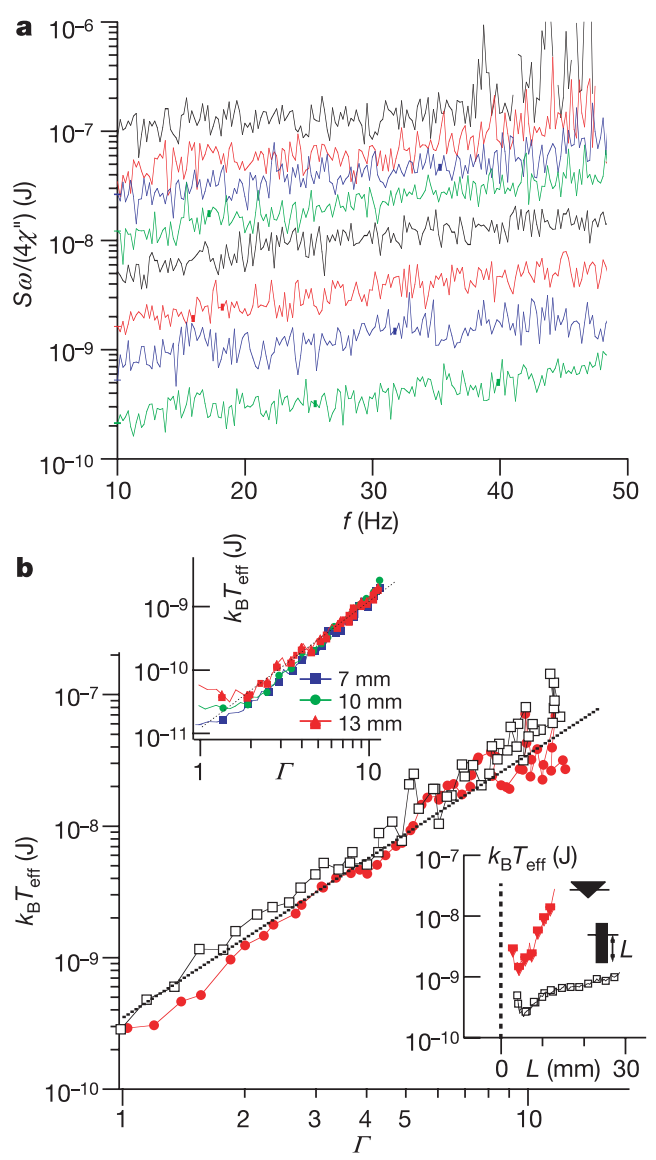

Figure $3 \mathrm{FD}$ ratio and the effective temperature. a, The FD ratio $S(\omega) \omega /\left(4 \chi^{\prime \prime}(\omega)\right)$, versus $f=\omega / 2 \pi$ for different vibration intensities $\Gamma$, obtained from Fig. 2 . The intensity values are, from top to bottom, 11.6, 10.0, 7.3, 5.4, 3.7, 2.2, 1.5 and 1.0. b. The FD ratio level (averaged from $10 \mathrm{~Hz}$ to $50 \mathrm{~Hz}$ ), that is, the effective temperature $T_{\text {eff, according to }}$ the expression $S(\omega) \omega /\left(4 \chi^{\prime \prime}(\omega)\right)=k_{\mathrm{B}} T_{\text {eff, }}$, versus $\Gamma$, from data in $\mathbf{a}$ (black open symbols), and from similar data obtained using a conical probe with triple moment of inertia (red symbols) and the same $L$. A power law fitted to the data gives $T_{\text {eff }} \propto \Gamma^{p}$, with $p=2.1$. The dashed line has equation $k_{\mathrm{B}} T_{\text {eff }}=3.5 \times 10^{-10} \Gamma^{2}$. The same FD ratio levels are measured by feeding the vibrator with a filtered white noise with a different central frequency $(800 \mathrm{~Hz}$, not shown). The FD ratio level also depends on the tribological properties of the granular material (especially rich for glass $\left.{ }^{16}\right)$. We have obtained reproducible data using aged (that is, exposed for a long time to the atmosphere) glass beads. Upper inset, the effective temperature $T_{\text {eff }}$ versus $\Gamma$, obtained using cylindrical probes of different diameters and polished surfaces. (For clarity, only one symbol every four data values is marked). The immersion depth is about $21 \mathrm{~mm}$. Notice the deviation from the $T_{\text {eff }} \propto \Gamma^{2}$ dependence at low $\Gamma$ (see text for details). Lower inset, the effective temperature $T_{\text {eff }}$ at $\Gamma=9$, versus the immersion depth of the oscillator, $L$, for conical (red symbols) and cylindrical (black symbols) probes (see text for details) (For clarity, only one symbol every four data values is marked). Notice that the precise position of the surface is unknown for a vibrated granular medium, but it is approximately at $L=0$ (vertical dashed line). The bottom of the container is at $L=31 \mathrm{~mm}$. We note that, owing to boundary effects, in the layer close to the surface (about $0<L<5 \mathrm{~mm}$ ), the FD ratio is no longer 'flat', and we do not extract an effective temperature in this region. 
of $\Gamma$. (2) While the granular medium is vibrated at a given intensity $\Gamma$, a sinusoidal torque $C_{\text {ext }}(t)=C_{\mathrm{e}} \sin (\omega t)$ is applied to the oscillator using a permanent magnet fixed on the oscillator and two external coils. The complex susceptibility at the given $\omega$ is obtained from $\chi(\omega)=\theta(\omega) / C_{\text {ext }}(\omega)$, where $\theta(\omega)$ and $C_{\text {ext }}(\omega)$ are the Fourier transforms of $\theta(t)$ and $C_{\text {ext }}(t)$, respectively. The amplitude $C_{\mathrm{e}}$ of the external torque is small enough to be in the regime of linear response, that is, we measure the linear susceptibility. The complex susceptibility $\chi(\omega)$ versus $\omega$ is obtained by sweeping the frequency of the sinusoidal torque. The modulus of the complex susceptibility, $|\chi(\omega)|$, for different intensities of vibration $\Gamma$, is shown in Fig. $2 b$.

With both the susceptibility and the noise PSD data, we are now able to check whether the analogy to the thermally induced brownian motion makes sense. First of all, the modulus of the complex susceptibility, $|\chi(\omega)|$, is fitted remarkably well by the susceptibility expression for the damped oscillator, $|\chi(\omega)|=$ $\left[I^{2}\left(\omega_{o}^{2}-\omega^{2}\right)^{2}+\alpha^{2} \omega^{2}\right]^{-1 / 2}$, as shown in Fig. 2b. This means that the deterministic equation of motion, that is, $I\left(\mathrm{~d}^{2} \theta / \mathrm{d} t^{2}\right)+$ $\alpha(\mathrm{d} \theta / \mathrm{d} t)+G \theta=C_{\text {ext }}(t)$, describes well the response of the immersed oscillator, and allows us to define a granular friction coefficient $\alpha$, or a granular viscosity $\mu \propto \alpha$. From the fitting parameters at different $\Gamma$ we also deduce that $\alpha \propto 1 / \Gamma$, as shown in the inset of Fig. $2 b$.

Second, we can obtain the FD ratio $S(\omega) \omega /\left(4 \chi^{\prime \prime}(\omega)\right)$ from our data. Figure 3a shows the FD ratio versus $f=\omega / 2 \pi$ at different $\Gamma$. The FD ratio is surprisingly 'flat', that is, approximately independent of the frequency in the observed low-frequency range (especially compared to what has been measured in other nonequilibrium thermal systems, such as in laponite ${ }^{7}$ and glycerol ${ }^{8}$.) This means that the high-frequency driven agitation of the granular medium acts on the oscillator as a source of random torque with white spectrum, at least in the $10-50 \mathrm{~Hz}$ range under investigation. Energy is thus injected at high-frequency, and spreads into a lowfrequency white spectrum. A roughly flat FD ratio also provides support for the existence of an FD relation in off-equilibrium driven granular steady states. The FD ratio level can thus be used to define an effective temperature, $T_{\text {eff. }}$. Figure $3 \mathrm{~b}$ shows the averaged $\mathrm{FD}$ ratio levels, that is, $k_{\mathrm{B}} T_{\text {eff, versus }} \Gamma$. Fitting to a power-law we obtain $k_{\mathrm{B}} T_{\text {eff }} \propto \Gamma^{p}$ with $p$ close to 2 , thus giving $T_{\text {eff }} \propto \Gamma^{2}$.

Obviously, this is only a first-order approximation. Close inspection of Fig. 3a apparently shows a small frequency dependence. However, a useful torsion signal is only detected around the natural frequency of the oscillator, limiting our accessible frequency range. In future work, the study of the frequency dependence of the FD ratio over a large frequency range could give insights about possible energy 'cascade' in vibro-fluidized granular matter. Notice also that a deviation from the $T_{\text {eff }} \propto \Gamma^{2}$ dependence is seen close to the lifton threshold, depending on the probe used (upper inset Fig. 3b). The main reason is that the immersed probe, which does not move in the vertical or horizontal directions, becomes a source of localized vibration at low $\Gamma$. In other words, at low $\Gamma$ the granular medium is 'warmer' around the probe than in the bulk (the 'thermometer heats the sample'), and the $\Gamma$ dependence of the effective temperature changes.

In the frame of this first-order approximation, our results suggest a simple picture. Owing to the complex dissipation processes between the grains, only a fixed fraction of the energy injected by the vibrator is effectively available as granular kinetic energy and is 'sensed' by the oscillator. In fact, we notice that the order of magnitude of the thermal energy, that is, $k_{\mathrm{B}} T_{\text {eff, }}$, as measured here, is consistent with realistic values of the mean kinetic energy per particle, as measured by grain-tracking methods (see, for example, refs 9 and 10). Thus, the effective temperature $T_{\text {eff }}$ we measure seems to be related to the granular temperature, as usually defined in granular gases ${ }^{5,6}$. Notice that the measured granular friction coefficient $\alpha$ decreases by increasing the effective temperature, since $\alpha \propto 1 / \Gamma$ and $T_{\text {eff }} \propto \Gamma^{2}$. This is the behaviour of liquids rather than gases. However, the oscillator sees an increasing effective temperature and a decreasing granular density as $\Gamma$ is increased. A similar effect has been reported by Zik et al. ${ }^{11}$ by observing the mobility of a sphere immersed in a vibro-fluidized granular medium.

We now study the dependence of $T_{\text {eff }}$ on the oscillator properties, as well as on the characteristics of the granular medium, such as immersion depth and granular anisotropy. Figure $3 \mathrm{~b}$ addresses all these issues. The main panel of Fig. 3b shows data obtained using a conical probe with different moments of inertia. The upper inset of Fig. 3 b shows data obtained using cylindrical probes with different diameters. In all these circumstances it turns out that $T_{\text {eff }}$ is insensitive to the change of these parameters. This is an indication that $T_{\text {eff }}$ could be an intrinsic property of the granular medium.

The bottom inset of Fig. 3b shows data measured at different immersion depths, using conical and cylindrical probes. Two important aspects need to be underlined: First, in a layer close to the surface the measured FD ratio shows a strong frequency dependence, and it becomes 'flat' (as in Fig. 3a) only at a sufficient immersion depth. (The FD ratio becomes 'flat' approximately at the depth where a minimum appears in the bottom inset of Fig. 3b). Underneath the surface layer, we observe that $T_{\text {eff }}$ increases with the depth. This is possibly related to a slight decrease of the granular density with the depth, as observed in simulations ${ }^{12}$ and measurements ${ }^{13}$. The behaviour of the surface layer itself needs to be explored more carefully, as we cannot exclude a relation with density induced features (as observed in refs 10, 12 and 13). A second point is the difference between $T_{\text {eff }}$ measured by conical and cylindrical probes. This is a peculiar granular effect and it is probably related to the intrinsic anisotropy of the system under gravity. Although the diameter of the horizontal section of the probe plays no role, the angle of inclination of the surface exposed to the granular agitation, relative to the vertical axis, seems to be a relevant parameter. As reported in ref. 10, the granular temperature is anisotropic, corresponding to horizontal and vertical impulse components. One thus expects in particular the transfer of the vertical impulse into torsion momentum to depend on the geometry and the surface state of the probe. Nevertheless, for a given kind of probe-for example, conical or cylindrical-the present experiment shows that one can satisfy the requirements for the Langevin approach to be applicable.

The most important finding obtained here is that it is possible to separate slow and fast degrees of freedom in vibro-fluidized granular matter and to model it, in the spirit of the Langevin approach, in terms of two intrinsic parameters: an effective friction coefficient and an effective temperature. In this perspective the oscillator indeed behaves as a brownian oscillator, and the vibro-fluidized granular matter can be seen as a macroscopic 'thermal bath'. We note that the analogy is only formal, since the granular medium is never in equilibrium in the thermodynamic sense. The experiment also shows that granular matter's mechanical anisotropy and inhomogeneity due to gravitation play an important role, inducing complications that are not present in usual gas or liquid media, which can be assumed to be isotropic and homogeneous.

Received 19 March; accepted 24 June 2003; doi:10.1038/nature01867.

\footnotetext{
1. Gerlach, W. \& Lehrer, E. Über die Messung der rotatorischen Brownschen Bewegung mit Hilfe einer Drehwage. Naturwissenschaften 15, 15 (1927).

2. Uhlenbeck, G. E. \& Goudsmit, S. A problem in Brownian motion. Phys. Rev. 34, 145-151 (1929).

3. Wang, M. C. \& Uhlenbeck, G. E. On the theory of the Brownian motion II. Rev. Mod. Phys. 17, 323-342 (1945).

4. Kubo, R., Toda, M. \& Hashitsume, N. Statistical Physics II: Nonequilibrium Statistical Mechanics (Springer, Berlin, 1991).

5. Jaeger, H. M., Nagel, S. R. \& Behringer, R. P. Granular solids, liquids and gases. Rev. Mod. Phys. 68, 1259-1273 (1996).

6. Mehta, A. \& Halsey, T. C. (eds) Proceedings of the Workshop "Challenges in Granular Physics". Adv. Complex Syst. 4 (special issue), 287-534 (2001).

7. Bellon, L., Ciliberto, S. \& Laroche, C. Violation of the fluctuation-dissipation relation during the formation of a colloidal glass. Europhys. Lett. 53, 511-517 (2001).
} 
8. Grigera, T. S. \& Israeloff, N. F Observation of a fluctuation-dissipation-theorem violation in a structural glass. Phys. Rev. Lett. 83, 5038-5041 (1999).

9. Dixon, P. K. \& Durian, D. J. Speckle visibility spectroscopy and variable granular fluidization. Phys Rev. Lett. 90, 184302 (2003)

10. Yang, X., Huan, C., Candela, D., Mair, R. W. \& Walsworth, R. L. Measurements of grain motion in dense, three-dimensional granular fluid. Phys. Rev. Lett. 88, 044301 (2002).

11. Zik, O., Stavans, J. \& Rabin, Y. Mobility of a sphere in vibrated granular media. Europhys. Lett. 17, 315-319 (1992)

12. Barrat, A. \& Loreto, V. Response properties in a model for granular matter. J. Phys. A 33, 4401-4426 (2000).

13. Philippe, P. \& Bideau, D. Compaction dynamics of a granular medium under vertical tapping Europhys. Lett. 60, 677-683 (2002).

14. D’Anna, G. \& Gremaud, G. Vogel-Fulcher-Tammann type diffusive slowdown in weakly perturbed granular media. Phys. Rev. Lett. 87, 254-302 (2001).

15. D’Anna, G., Mayor, P., Gremaud, G., Barrat, A. \& Loreto, V. Extreme events-driven glassy behaviour in granular media. Europhys. Lett. 61, 60-66 (2003).

16. Olivi-Tran, N., Fraysse, N., Girard, P., Ramonda, M. \& Chatain, D. Modeling and simulations of the behavior of glass particles in a rotating drum in heptane and water vapor atmospheres. Eur. Phys. J. B 25, 217-222 (2002).

Acknowledgements We thank Z. Racz and A. Vespignani for discussions and comments. A.B. and V.L. wish to thank the Ecole Polytechnique Fédérale de Lausanne for its hospitality during the realization of this work. F.B. thanks the National Science Foundation for support.

Competing interests statement The authors declare that they have no competing financial interests.

Correspondence and requests for materials should be addressed to G.D. (gianfranco.danna@epfl.ch).

\section{Signature of optimal doping in Hall-effect measurements on a high-temperature superconductor}

Fedor F. Balakirev ${ }^{1}$, Jonathan B. Betts ${ }^{1}$, Albert Migliori ${ }^{1}$, S. Ono ${ }^{2}$, Yoichi Ando ${ }^{2}$ \& Gregory S. Boebinger

${ }^{1}$ National High Magnetic Field Laboratory, Los Alamos National Laboratory, Los Alamos, New Mexico 87545, USA

${ }^{2}$ Central Research Institute of Electric Power Industry, Komae, Tokyo 201-8511, Japan

High-temperature superconductivity is achieved by doping copper oxide insulators with charge carriers. The density of carriers in conducting materials can be determined from measurements of the Hall voltage-the voltage transverse to the flow of the electrical current that is proportional to an applied magnetic field. In common metals, this proportionality (the Hall coefficient) is robustly temperature independent. This is in marked contrast to the behaviour seen in high-temperature superconductors when in the 'normal' (resistive) state $^{1-5}$; the departure from expected behaviour is a key signature of the unconventional nature of the normal state, the origin of which remains a central controversy in condensed matter physics ${ }^{6}$. Here we report the evolution of the low-temperature Hall coefficient in the normal state as the carrier density is increased, from the onset of superconductivity and beyond (where superconductivity has been suppressed by a magnetic field). Surprisingly, the Hall coefficient does not vary monotonically with doping but rather exhibits a sharp change at the optimal doping level for superconductivity. This observation supports the idea that two competing ground states underlie the high-temperature superconducting phase.

In the 16 years since the discovery of high-temperature superconductors, much has been learned about the many unusual properties of these materials. Most prominently, the high-temperature superconducting state has been shown to be the first known example of ' $d$-wave' superconductivity, in which the superconducting energy gap has the symmetry of a $d_{x^{2}-y^{2}}$ atomic orbital. The underlying mechanism that gives rise to this $d$-wave superconductivity is less well established. There is increasing evidence that the key role is played by an antiferromagnetic phase, in which neighbouring electron spins are anti-aligned, that is in competition with superconductivity. There remains, however, a significant lack of understanding about the 'normal-state' behaviour of the hightemperature superconductors, behaviour that is so consistently unusual that many believe the most promising route to a complete understanding of high-temperature superconductivity lies in an understanding of this abnormal 'normal state'. Among the various abnormal normal-state properties, the Hall effect has been notoriously difficult to understand.

The properties of $\mathrm{Bi}_{2} \mathrm{Sr}_{2-x} \mathrm{La}_{x} \mathrm{CuO}_{6+\delta}$ (BSLCO) make it an ideal compound for a comprehensive study of the normal state at low temperatures. High-quality BSLCO single crystals can be produced over a wide range of carrier concentration by partially substituting $\mathrm{Sr}$ with La (ref. 7). Most importantly for these studies, a 60-T magnetic field, currently the practical limit for non-destructive generation of magnetic fields, is sufficient to completely suppress the superconductivity in BSLCO $^{8}$. Single-crystal samples of BSLCO were prepared using the floating-zone technique ${ }^{7}$, and were exceptionally well characterized by measurements of resistivity, hightemperature Hall effect and thermopower among other techniques $^{5,7-11}$.

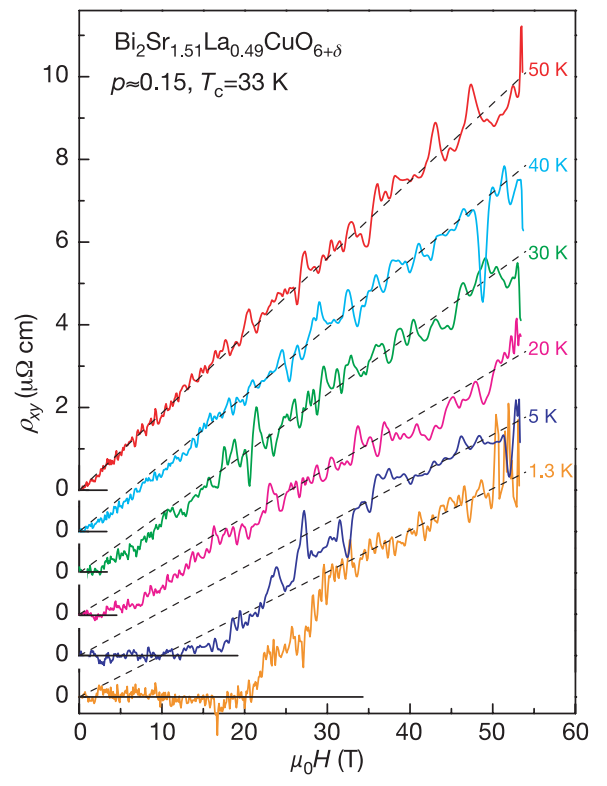

Figure 1 Hall resistivity versus magnetic field. Shown here is a typical Hall signal in a $\mathrm{Bi}_{2} \mathrm{Sr}_{0.51} \mathrm{La}_{0.49} \mathrm{CuO}_{6+\delta}$ superconducting sample for which $T_{\mathrm{c}}=33 \mathrm{~K}$ and the hole doping $p \approx 0.15$. Measurements of the Hall resistivity were carried out during a magnetic field pulse of $\sim 100 \mathrm{~ms}$ duration using a standard six-probe geometry for a series of ten samples. The data traces were recorded on a computer using a high-resolution low-noise synchronous lock-in technique developed at the National High Magnetic Field Laboratory. Data are shown for temperatures both above and below $T_{\mathrm{c}}$, and the traces are offset vertically for clarity. The Hall resistivity above $T_{\mathrm{C}}$ shows the conventional linear magneticfield dependence at all magnetic fields up to $55 \mathrm{~T}$. At temperatures substantially below $T_{\mathrm{C}}$, the Hall resistivity is zero at low magnetic field owing to the presence of the superconducting phase in the sample. As the magnetic field suppresses superconductivity, the Hall signal rapidly increases and then recovers its conventional linear-in-magnetic-field behaviour. The dashed lines represent best linear fit,

$\rho_{X y}(H)=R_{H} H$, for the high-field regime in which superconductivity is suppressed by magnetic field, where $R_{H}$ is the Hall coefficient, which, in a simple metal, is inversely proportional to the number of charge carriers in the material. 